\title{
Effect of Different Weed Management Practices on Growth and Yield in Soybean Pigeonpea Intercropping System and its Residual Effect on Succeeding Crops
}

\author{
R. V. Hajari ${ }^{{ }^{*}}$ and G. J. Patel ${ }^{2}$ \\ ${ }^{1}$ Department of Agronomy, B.A. College of Agriculture, Anand Agricultural University, \\ Anand- 388110 Gujarat (India) \\ ${ }^{2}$ Tribal Research cum Training Center, Anand Agricultural University, \\ Devgadhbaria - 389380 Anand-388110 Gujarat (India) \\ *Corresponding author
}

\section{A B S T R A C T}

\begin{tabular}{|l|}
\hline K e y w o r d s \\
$\begin{array}{l}\text { Hand weeding, } \\
\text { Herbicide and } \\
\text { soybean equivalent } \\
\text { yield }\end{array}$ \\
\hline Article Info \\
\hline $\begin{array}{l}\text { Accepted: } \\
\text { 17 August } 2020 \\
\text { Available Online: } \\
\text { 10 September } 2020\end{array}$ \\
\hline
\end{tabular}

\section{Introduction}

Soybean considered as the 'Wonder legume'. It has outstanding nutritive value with $43 \%$ biological protein, $20 \%$ oil and is also very rich in vitamins, iron, mineral, salts and amino acids (Sangeetha et al., 2013). Pigeonpea (Cajanus cajan (L.) Millsp.) commonly known as red gram, tur or arhar is the fifth prominent legume crop in the world. It has been recognized as a valuable source of protein for the vegetarians in their daily diet.
The field experiment was conducted at Agriculture Research Station, Anand Agricultural University, Derol, Dist: panchmahal, Gujarat, India during kharif seasons of the years 2017-18 and 2018-19. Ten treatments for weed management were studied in randomized block design with four replications to study the effect of different weed management practices on growth and yield in soybean - pigeonpea intercropping system and its residual effect on succeeding crops. Result of field experimentation, weeds could be managed by adopting weed management practices interculturing + hand weeding at $20 \& 40$ DAS or post - emergence application of imazamox 35\% + imazethapyr 35\% (Pre mix)70 $\mathrm{g} \mathrm{ha}^{-}$ ${ }^{1} \mathrm{PoE} f b$ interculturing + hand weeding at $30 \mathrm{DAS}$ was recorded higher growth, growth and yield attributes, soybean equivalent yield, gross returns, net returns, benefit cost ratio and none of the herbicides showed adverse effect on succeeding crops viz., maize, pearl millet and cucumber grown after harvest of soybean and pigeonpea without disturbing soil.
It is grown as sole crop or intercrop with urdbean, mungbean, castor, sorghum, soybean, cotton, maize and groundnut in different states like Maharashtra, Karnataka, Andhra Pradesh, Gujarat, Jharkhand, Rajasthan Odisha, Punjab and Haryana. In rainy season weeds come in 2-3 flushes and growth is very fast therefore, they compete for light, nutrient, and space and are responsible for considerable reduction in yield. Weeds caused $80 \%$ reduction in pigeonpea grain yield if weeds were allowed 
to grow till harvest, however, grain yield losses were only $38 \%$ in pigeonpea + soybean intercropping system (Talnikar et al., 2008).

Intercropping is the agricultural practice of growing two or more crops simultaneously on the same field with a definite row proportion. Weed suppression, the reduction of weed growth by crop interference, has been referred as one determinant of yield advantage of intercropping. The first 20-40 days period after sowing of soybean is considered to be critical with respect to weed crop competition. Weed competition during this period may lead to $40-70 \%$ reduction in the seed yield of soybean (Bhan, 1975) depending upon the weed species present.

Weeds are known to cause 40-64\% reduction in pigeonpea yield. The critical period of crop weed competition for pigeonpea is 4-8 weeks (Ali, 1992) ${ }^{[1]}$. Among various constraints in crop production weed control is important one but generally neglected by many farmers.

The crop yield loss is 20 to 77 per cent due to weeds (Kurchania et al., 2001). Inter cropping suppress the growth of weeds up to $25 \%$ (Sobney et al., 1989). It is very essential to find out alternative to manual labour for weed control, which has more weed control efficiency. At present many promising and selective herbicides are available which can control weeds effectively. Therefore, the present study was conducted to study the effect of different weed management practices on growth and yield in soybean-pigeonpea intercropping system and its residual effect on succeeding crops. Herbicides proved important major in modern agriculture practices. With the intense farming practice and multiple cropping, the paucity of agricultural labour is seen. Their peak demand for a short period, inaccessible field conditions during crop seasons also encourages using herbicides in modern farming. But continuous application of herbicides in intence cropping system may leads to its residue accumulation in the soil. This persistence of an herbicide may limit the choice of succeeding crops. Therefore, residual studies essential to determine the feasibility of any herbicide. Summer maize, pearl millet and cucumber are important succeeding crops grown after soybeanpigeonpea intercrops in middle Gujarat region. Keeping this in view the present experiment is proposed.

\section{Materials and Methods}

Field experiment was carried out to study the effect of different weed management practices on growth and yield in soybean - pigeonpea intercropping system and its residual effect on succeeding crops The experiment was conducted at Agriculture Research Station, Anand Agricultural University, Derol, Dist: Panchmahal, Gujarat, India during kharif seasons of the years 2017-18 and 2018-19. The ten weed control treatments were studied viz., pendimethalin $1000 \mathrm{~g} \quad \mathrm{ha}^{-1} \mathrm{PE} \quad f b$ interculturing + hand weeding at 30 DAS, clomazone $1000 \mathrm{~g} \mathrm{ha}^{-1} \mathrm{PE} f b$ interculturing + hand weeding at 30 DAS, imazethapyr $75 \mathrm{~g}$ $\mathrm{ha}^{-1} \mathrm{PoE} f b$ interculturing + hand weeding at 30 DAS, propaquizafop $75 \mathrm{~g} \mathrm{ha}^{-1}$ PoE $f b$ interculturing + hand weeding at 30 DAS, quizalofop ethyl $50 \mathrm{~g}$ ha $^{-1}$ PoE $f b$ interculturing + hand weeding at 30 DAS, fenoxaprop-p-ethyl $100 \mathrm{~g} \mathrm{ha}^{-1}$ PoE $f b$ interculturing + hand weeding at 30 DAS, imazamox 35\% + imazethapyr 35\% (Pre mix) $70 \mathrm{~g} \mathrm{ha}^{-1} \mathrm{PoE} f b$ interculturing + hand weeding at 30 DAS, sodium acefluorfen $16.5 \%+$ clodinafop propargyl 8\% EC (Pre-mix) $80+165$ PoE $f b$ interculturing + hand weeding at $30 \mathrm{DAS}$, interculturing + hand weeding at $20 \& 40$ DAS and weedy check. The experiment was laid out in Randomized Block design with four replications. The plot size was $3.6 \times 6.0 \mathrm{~m}$. The soil of the experimental field was sandy loam in texture having low in 
organic carbon and nitrogen, medium in available phosphorus and high in potassium. Pendimethalin and clomazonewas applied as pre emergence while imazethapyr, propaquizafop, quizalofop ethyl, fenoxapropp-ethyl, imazamox $35 \%+$ imazethapyr $35 \%$ and sodium acefluorfen $16.5 \%+$ clodinafop propargyl $8 \%$ EC were applied as post emergence with flat fan nozzle using 500 litre water per hectare. The soybean variety NRC 37 and pigeonpea variety AGT 2 were used in the experiment as a test crop. After harvesting of the soybean and pigeonpea crop the indicator plants was grown for bioassay study (up to 30 DAS). Three crops viz., maize, pearl millet and cucumber was grown in different row with minimum disturbance of the soil. The indicator plants was grown per plot two row of each crop for seed are sown maize (60 dibbles), pearl millet (120 dibbles) and cucumber (40 dibbles) in between the two row of previous crop pigeonpea stubbles without disturbance. All the recommended agronomic practices were followed for raising the crop during both the years of experimentation. The observations on growth and yield attributes were recorded. Observations on bio-assay study (germination (percent) at $10 \mathrm{DAS}$, plant height/shoot length, dry matter accumulation and root length of plant at 30 DAS) were recorded. Pooled analysis of two years was done and comparison was made at $5 \%$ level of significance.

\section{Results and Discussion}

\section{Effects on growth and yield attributes}

\section{Plant height (cm)}

The plant height at harvest of soybean is presented in (Table 1). Significantly higher plant height of soybean was recorded at harvest (74.9) by the treatment of $\mathrm{T}_{9}$ (IC + HW at $20 \& 40$ DAS). Significantly lower plant height was observed under treatment $T_{10}$ (weedy check). Plant height of pigeonpea was recorded at harvest as were not affected significantly due to different weed control treatments (Table 1). The higher value of weed control treatment may be assigned to the extent of crop weed competition significantly maximum plant height all successive stages because the interculturing + hand weeding at $20 \& 40$ DAS provided weed free condition at critical stage of crop weed competition and created favorable condition for growth parameters. Similar, result was obtained by Upadhyay et al., (2012) in soybean and Jadhav (2015) in soybean + pigeonpea intercropping.

\section{Branches plant ${ }^{-1}$}

Significantly more number of branches plant ${ }^{-1}$ soybean (3.8) and pigeonpea (10.2) was recorded at harvest under $\mathrm{T}_{9}$ (IC $+\mathrm{HW}$ at 20 \& 40 DAS), which was at par with treatment $\mathrm{T}_{7}$ (imazamox 35\% + imazethapyr 35\% (Pre mix) $70 \mathrm{~g} \mathrm{ha}^{-1} \mathrm{PoE} f b \mathrm{IC}+\mathrm{HW}$ at $\left.30 \mathrm{DAS}\right)$ at 30 DAS (3.6) in soybean, while imazamox $35 \%$ + imazethapyr 35\% (Pre mix) $70 \mathrm{~g} \mathrm{ha}^{-}$ ${ }^{1} \mathrm{PoE} f b$ interculturing + hand weeding at 30 DAS (9.9), imazethapyr $75 \mathrm{~g}$ ha $^{-1} \mathrm{PoE} f b$ interculturing + hand weeding at 30 DAS (9.8), propaquizafop $75 \mathrm{~g} \mathrm{ha}^{-1}$ PoE $f b$ interculturing + hand weeding at 30 DAS (9.7), sodium acefluorfen $16.5 \%$ + clodinafop propargyl 8\% EC (Pre mix) 80+165 PoE $f b$ interculturing + hand weeding at 30 DAS (9.4) and quizalofop ethyl $50 \mathrm{~g} \mathrm{ha}^{-1} \mathrm{PoE} f b$ interculturing + hand weeding at 30 DAS (9.3) in pigeonpea. Significantly less number of branches plant ${ }^{-1}$ (soybean (2.0) and pigeonpea (6.5) was recorded by the treatment $\mathrm{T}_{10}$ (weedy check) (Table 1).

Similar, results were obtained by Upadhyay et al., (2012) in soybean and Jadhav (2015) in soybean + pigeonpea intercropping. 
Table.1 Growth and yield attributes as influenced by weed management practices in soybean-pigeonpea intercropping system (pooled of two years)

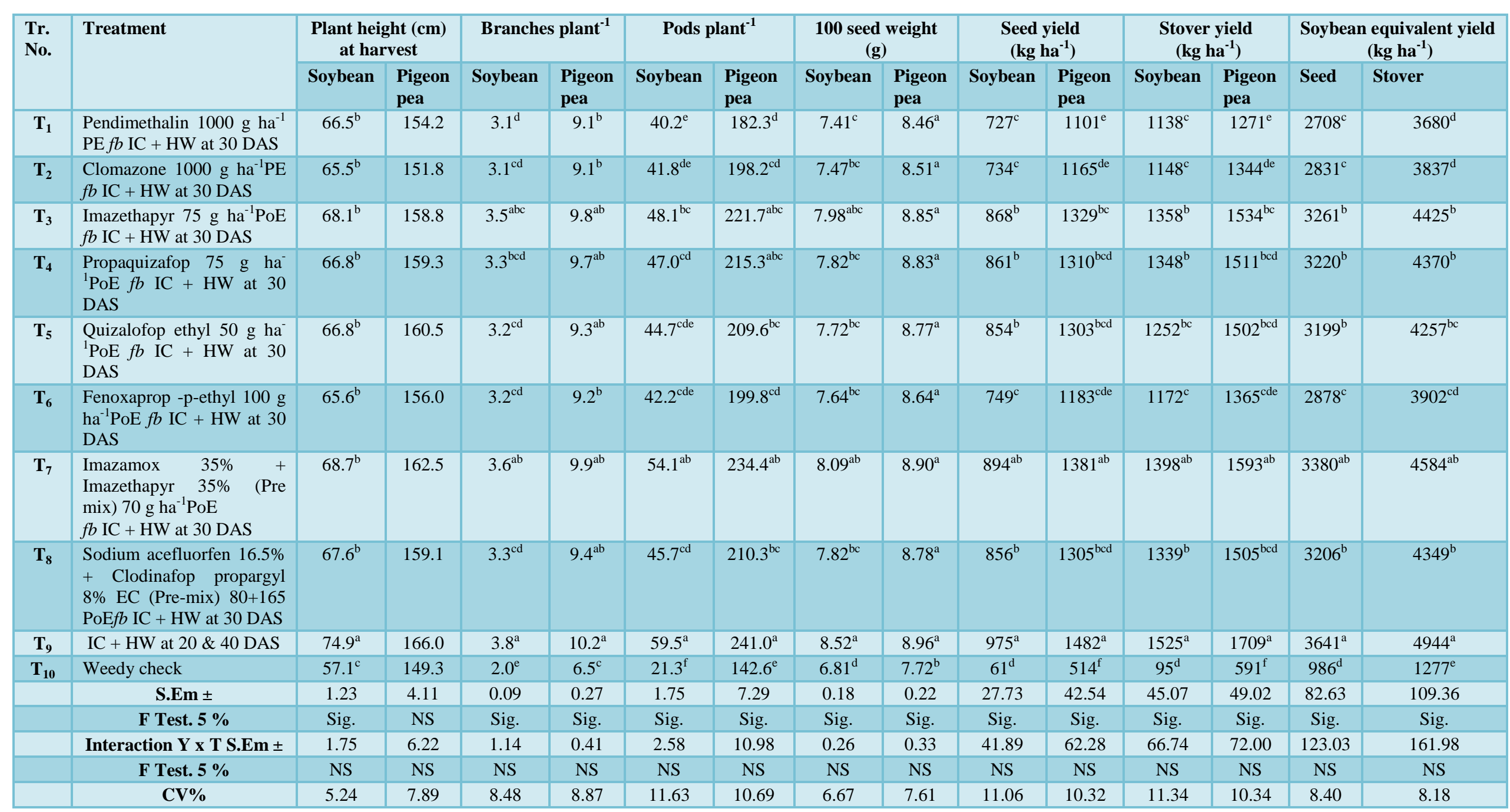


Table.2 Economics as influenced by weed management practices in soybean-pigeonpea intercropping system (pooled of two years)

\begin{tabular}{|c|c|c|c|c|c|c|c|}
\hline \multirow{2}{*}{$\begin{array}{l}\text { Tr. } \\
\text { No. }\end{array}$} & \multirow[t]{2}{*}{ Treatment } & \multicolumn{2}{|c|}{ Soybean equivalent yield } & \multirow{2}{*}{$\begin{array}{l}\text { Gross } \\
\text { return } \\
\left(\text { ha }^{-1}\right)\end{array}$} & \multirow{2}{*}{$\begin{array}{l}\text { Cost of } \\
\text { cultivation } \\
\left(\text { ha }^{-1}\right)\end{array}$} & \multirow{2}{*}{$\begin{array}{l}\text { Net return } \\
\left(\text { ha }^{-1}\right)\end{array}$} & \multirow[t]{2}{*}{$\mathrm{B}: \mathrm{C}$ ratio } \\
\hline & & $\begin{array}{l}\text { Seed } \\
\text { Yield } \\
\left(\mathrm{kg} \mathrm{ha}^{-1}\right)\end{array}$ & $\begin{array}{l}\text { Stover } \\
\text { Yield } \\
\left(\mathrm{kg} \mathrm{ha}^{-1}\right)\end{array}$ & & & & \\
\hline $\mathbf{T}_{1}$ & $\begin{array}{l}\text { Pendimethalin } 1000 \mathrm{~g} \mathrm{ha}^{-1} \mathrm{PE} f b \mathrm{IC}+ \\
\mathrm{HW} \text { at } 30 \mathrm{DAS}\end{array}$ & $2708^{c}$ & $3680^{\mathrm{d}}$ & 84931 & 49525 & 35406 & 1.71 \\
\hline $\mathbf{T}_{2}$ & $\begin{array}{l}\text { Clomazone } 1000 \mathrm{~g} \mathrm{ha}^{-1} \mathrm{PE} f b \mathrm{IC}+\mathrm{HW} \\
\text { at } 30 \mathrm{DAS}\end{array}$ & $2831^{\mathrm{c}}$ & $3837^{\mathrm{d}}$ & 88779 & 49232 & 39547 & 1.80 \\
\hline $\mathbf{T}_{\mathbf{3}}$ & $\begin{array}{l}\text { Imazethapyr } 75 \mathrm{~g} \mathrm{ha}^{-1} \mathrm{PoE} f b \mathrm{IC}+\mathrm{HW} \\
\text { at } 30 \mathrm{DAS}\end{array}$ & $3261^{b}$ & $4425^{b}$ & 102248 & 48908 & 53340 & 2.09 \\
\hline $\mathbf{T}_{4}$ & $\begin{array}{l}\text { Propaquizafop } 75 \mathrm{~g} \mathrm{ha}^{-1} \mathrm{PoE} f b \mathrm{IC}+ \\
\text { HW at } 30 \text { DAS }\end{array}$ & $3220^{b}$ & $4370^{b}$ & 100959 & 48849 & 52110 & 2.07 \\
\hline $\mathbf{T}_{5}$ & $\begin{array}{l}\text { Quizalofop ethyl } 50 \mathrm{~g} \mathrm{ha}^{-1} \mathrm{PoE} f b \mathrm{IC}+ \\
\mathrm{HW} \text { at } 30 \text { DAS }\end{array}$ & $3199^{b}$ & $4257^{b c}$ & 100238 & 49444 & 50794 & 2.03 \\
\hline $\mathbf{T}_{6}$ & $\begin{array}{l}\text { Fenoxaprop -p-ethyl } 100 \mathrm{~g} \mathrm{ha}^{-1} \mathrm{PoE} f b \\
\mathrm{IC}+\mathrm{HW} \text { at } 30 \text { DAS }\end{array}$ & $2878^{c}$ & $3902^{\mathrm{cd}}$ & 90245 & 48816 & 41429 & 1.85 \\
\hline $\mathbf{T}_{7}$ & $\begin{array}{l}\text { Imazamox } 35 \%+\text { Imazethapyr } 35 \% \\
\text { (Pre mix) } 70 \mathrm{~g} \mathrm{ha}^{-1} \mathrm{PoE} f b \mathrm{IC}+\mathrm{HW} \text { at } \\
\text { 30 DAS }\end{array}$ & $3380^{\mathrm{ab}}$ & $4584^{\mathrm{ab}}$ & 105971 & 49620 & 56351 & 2.14 \\
\hline $\mathbf{T}_{8}$ & $\begin{array}{l}\text { Sodium acefluorfen } 16.5 \%+ \\
\text { Clodinafop propargyl } 8 \% \text { EC (Pre- } \\
\text { mix) } 80+165 \text { PoE } f b \text { IC }+ \text { HW at } 30 \\
\text { DAS }\end{array}$ & $3206^{b}$ & $4349^{b}$ & 100520 & 49931 & 50589 & 2.01 \\
\hline $\mathbf{T}_{9}$ & $\mathrm{IC}+\mathrm{HW}$ at $20 \& 40 \mathrm{DAS}$ & $3641^{\mathrm{a}}$ & $4944^{\mathrm{a}}$ & 114186 & 54187 & 59999 & 2.11 \\
\hline $\mathbf{T}_{10}$ & Weedy check & $986^{\mathrm{d}}$ & $1277^{\mathrm{e}}$ & 30844 & 41151 & -10307 & 0.75 \\
\hline
\end{tabular}

\begin{tabular}{|l|l|}
\hline Selling Price & \\
\hline Seed: Soybean: ` $\mathbf{3 0 . 0} \mathbf{~ k g}^{-1}$ & Stover: Soybean: ` $1.0 \mathrm{~kg}^{-1}$ \\
\hline Pigeonpea:` $\mathbf{5 4 . 0} \mathbf{k g}^{-1}$ & Pigeonpea:`2.0 $\mathrm{kg}^{-1}$ \\
\hline
\end{tabular}


Table.3 Residual effect of weed management practices in succeeding crops (pooled of two years)

\begin{tabular}{|c|c|c|c|c|c|c|c|c|c|c|c|c|c|}
\hline \multirow[t]{2}{*}{$\begin{array}{l}\text { Tr. } \\
\text { No. }\end{array}$} & \multirow[t]{2}{*}{ Treatment } & \multicolumn{3}{|c|}{ Germination (\%) at 10 DAS } & \multicolumn{3}{|c|}{$\begin{array}{c}\text { Plant height/ Shoot length }(\mathrm{cm}) \\
\text { at } 30 \text { DAS }\end{array}$} & \multicolumn{3}{|c|}{$\begin{array}{l}\text { Dry matter accumulation (g } \\
\text { plant }^{-1} \text { ) at } 30 \text { DAS }\end{array}$} & \multicolumn{3}{|c|}{$\begin{array}{c}\text { Root length }(\mathrm{cm}) \text { at } \\
\text { 30 DAS }\end{array}$} \\
\hline & & Maize & $\begin{array}{l}\text { Pearl } \\
\text { millet }\end{array}$ & Cucumber & Maize & $\begin{array}{l}\text { Pearl } \\
\text { millet }\end{array}$ & Cucumber & Maize & $\begin{array}{l}\text { Pearl } \\
\text { millet }\end{array}$ & Cucumber & Maize & $\begin{array}{l}\text { Pearl } \\
\text { millet }\end{array}$ & Cucumber \\
\hline $\mathbf{T}_{1}$ & $\begin{array}{l}\text { Pendimethalin } 1000 \mathrm{~g} \mathrm{ha}^{-1} \mathrm{PE} \\
f b \mathrm{IC}+\mathrm{HW} \text { at } 30 \mathrm{DAS}\end{array}$ & 92.08 & 87.19 & 86.25 & 48.2 & 50.6 & 4.5 & 1.04 & 1.54 & 0.21 & 16.81 & 16.2 & 8.5 \\
\hline $\mathbf{T}_{2}$ & $\begin{array}{l}\text { Clomazone } 1000 \mathrm{~g} \mathrm{ha}^{-1} \mathrm{PE} f b \\
\mathrm{IC}+\mathrm{HW} \text { at } 30 \mathrm{DAS}\end{array}$ & 91.67 & 84.06 & 81.88 & 47.2 & 49.6 & 4.2 & 1.03 & 1.50 & 0.20 & 17.18 & 16.1 & 8.0 \\
\hline $\mathbf{T}_{\mathbf{3}}$ & $\begin{array}{l}\text { Imazethapyr } 75 \mathrm{~g} \mathrm{ha}^{-1} \mathrm{PoE} f b \\
\mathrm{IC}+\mathrm{HW} \text { at } 30 \mathrm{DAS}\end{array}$ & 94.38 & 83.44 & 84.69 & 48.9 & 46.9 & 4.6 & 1.06 & 1.49 & 0.21 & 16.87 & 16.0 & 7.4 \\
\hline $\mathbf{T}_{4}$ & $\begin{array}{l}\text { Propaquizafop } 75 \mathrm{~g} \mathrm{ha}^{-1} \mathrm{PoE} f b \\
\mathrm{IC}+\mathrm{HW} \text { at } 30 \mathrm{DAS}\end{array}$ & 86.88 & 81.88 & 81.88 & 48.0 & 51.1 & 4.4 & 0.98 & 1.47 & 0.20 & 15.42 & 15.8 & 8.3 \\
\hline $\mathbf{T}_{5}$ & $\begin{array}{l}\text { Quizalofop ethyl } 50 \mathrm{~g} \text { ha }^{-1} \mathrm{PoE} \\
f b \mathrm{IC}+\mathrm{HW} \text { at } 30 \mathrm{DAS}\end{array}$ & 91.25 & 82.40 & 82.50 & 48.8 & 50.2 & 4.6 & 1.03 & 1.47 & 0.21 & 17.76 & 16.6 & 8.2 \\
\hline $\mathbf{T}_{6}$ & $\begin{array}{l}\text { Fenoxaprop -p-ethyl } 100 \mathrm{~g} \text { ha } \\
{ }^{1} \mathrm{PoE} f b \text { IC + HW at } 30 \text { DAS }\end{array}$ & 88.54 & 88.23 & 80.00 & 44.3 & 47.1 & 4.4 & 1.00 & 1.58 & 0.20 & 16.67 & 17.0 & 8.5 \\
\hline $\mathbf{T}_{7}$ & 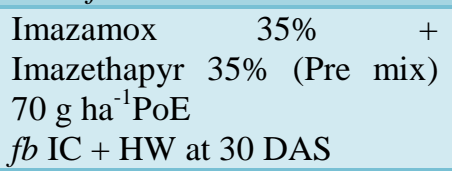 & 93.33 & 84.59 & 82.50 & 48.1 & 49.7 & 4.4 & 1.05 & 1.51 & 0.20 & 16.94 & 16.4 & 8.1 \\
\hline $\mathbf{T}_{8}$ & $\begin{array}{l}\text { Sodium acefluorfen } 16.5 \%+ \\
\text { Clodinafop propargyl } 8 \% \text { EC } \\
\text { (Pre-mix) } 80+165 \text { PoE } \\
f b \text { IC }+ \text { HW at } 30 \text { DAS }\end{array}$ & 92.08 & 84.27 & 83.13 & 48.8 & 47.6 & 4.6 & 1.04 & 1.51 & 0.21 & 16.41 & 16.2 & 8.2 \\
\hline $\mathbf{T}_{9}$ & $\mathrm{IC}+\mathrm{HW}$ at $20 \& 40 \mathrm{DAS}$ & 94.70 & 87.40 & 87.81 & 48.7 & 48.0 & 4.8 & 1.07 & 1.56 & 0.22 & 17.73 & 17.8 & 9.0 \\
\hline $\mathbf{T}_{10}$ & Weedy check & 91.67 & 86.46 & 80.00 & 48.6 & 50.1 & 4.5 & 1.03 & 1.55 & 0.20 & 16.81 & 16.6 & 7.7 \\
\hline & S.Em \pm & 2.47 & 2.16 & 2.05 & 1.47 & 1.67 & 0.16 & 0.02 & 0.04 & 0.01 & 0.68 & 0.68 & 0.38 \\
\hline & F Test. $5 \%$ & NS & NS & NS & NS & NS & NS & NS & NS & NS & NS & NS & NS \\
\hline & Interaction Y x T S.Em \pm & 3.49 & 3.17 & 3.08 & 2.14 & 2.49 & 0.23 & 0.04 & 0.06 & 0.01 & 0.99 & 1.04 & 0.56 \\
\hline & F Test. $5 \%$ & NS & NS & NS & NS & NS & NS & NS & NS & NS & NS & NS & NS \\
\hline & CV\% & 7.62 & 7.46 & 7.41 & 8.95 & 10.2 & 10.38 & 7.64 & 7.62 & 7.40 & 11.85 & 12.58 & 13.67 \\
\hline
\end{tabular}




\section{Pods plant ${ }^{-1}$}

Data regarding number of pods plant $^{-1}$ of soybean and pigeonpea was recorded at harvest as influenced by weed management practices are presented in (Table 1). Significantly higher number of pods plant ${ }^{-1}$ (59.5 and 241.0) of soybean and pigeonpea was recorded by the treatment $\mathrm{T}_{9}$ (IC $+\mathrm{HW}$ at $20 \& 40$ DAS), which was statistically at par with treatment $\mathrm{T}_{7}$ (imazamox $35 \%+$ imazethapyr 35\% (Pre mix) $70 \mathrm{~g} \mathrm{ha}^{-1}$ PoE $f b$ IC + HW at 30 DAS) (54.1) except all other treatments in soybean, while which was statistically at par with treatment $\mathrm{T}_{7}$ (imazamox 35\% + imazethapyr 35\% (Pre mix) $70 \mathrm{~g} \mathrm{ha}^{-1} \mathrm{PoE} f b \mathrm{IC}+\mathrm{HW}$ at $30 \mathrm{DAS}$ ) (234.4) and $\mathrm{T}_{3}$ (imazethapyr $75 \mathrm{~g} \mathrm{ha}^{-1} \mathrm{PoE} f b$ IC + HW at 30 DAS) (221.7) except all other treatments in pigeonpea. Significantly lower number of pods plant ${ }^{-1}$ (21.3 and 142.6) of soybean and pigeonpea was recorded by the treatment $\mathrm{T}_{10}$ (weedy check). Response of pods bearing capacity of plant to various treatments was significant. The treatment could very well be explained in the light of competition stress and clean cultivation. In soybean and pigeonpea, maximum number of pods were recorded in treatment interculturing + hand weeding at $20 \& 40$ DAS followed by treatment imazamox 35\% + imazethapyr 35\% (Pre mix) $70 \mathrm{~g} \mathrm{ha}^{-1}$ PoE $f b$ IC $+\mathrm{HW}$ at 30 DAS, The lowest number of pods per plant was recorded in weedy check. Similar result was reported by Mishra et al., (2013) and Upadhyay et al., (2012) in soybean.

\section{0 seed weight $(\mathrm{g})$}

Significantly higher number of seed weight (8.52) of soybean was recorded by the treatment $\mathrm{T}_{9}$ (IC $+\mathrm{HW}$ at $20 \& 40 \mathrm{DAS}$ ), which was statistically at par with treatment $\mathrm{T}_{7}$ (imazamox 35\% + imazethapyr 35\% (Pre mix) $70 \mathrm{~g} \mathrm{ha}^{-1} \mathrm{PoE} f b \mathrm{IC}+\mathrm{HW}$ at 30 DAS) (8.09) except all other treatments.
Significantly lowest seed weight $(6.81 \mathrm{~g})$ was recorded by the treatment $\mathrm{T}_{10}$ (weedy check). Similar result was opined by Prachand et al., (2014) in soybean. Significantly higher number of seed weight (8.96) was recorded by the treatment $\mathrm{T}_{9}$ (IC $+\mathrm{HW}$ at $20 \& 40$ DAS), which was statistically at par with all other treatments except treatment $\mathrm{T}_{10}$ (weedy check) (7.72) (Table 1). Similar result was opined by Rao et al., (2015) and Reddy et al., (2016) in pigeonpea.

\section{Seed yield $\left(\mathrm{kg} \mathrm{ha}^{-1}\right)$}

Data regarding seed yield $\left(\mathrm{kg} \mathrm{ha}^{-1}\right)$ of soybean and pigeonpea was recorded as influenced by weed management practices in pooled are presented in (Table 1). The higher seed yield of soybean and pigeonpea (975 and $1482 \mathrm{~kg}$ $\mathrm{ha}^{-1}$ ) was recorded under treatment $\mathrm{T}_{9}$ (IC + HW at $20 \& 40$ DAS), found statistically at par with treatment $\mathrm{T}_{7}$ (imazamox $35 \%+$ imazethapyr 35\% (Pre mix) $70 \mathrm{~g} \mathrm{ha}^{-1} \mathrm{PoE} f b$ IC + HW at 30 DAS). Significantly lowest seed yield of soybean and pigeonpea (61and $514 \mathrm{~kg} \mathrm{ha}^{-1}$ ) was recorded by the treatment $\mathrm{T}_{10}$ (weedy check).

Seed yield is an important parameter, which decides the efficiency superiority or stability of a particular treatment over treatments. The data of present investigation reveals that all weed control treatments, produced significantly higher seed yield over weedy check. Weed management due to different treatments at optimum time in the season, reduced crop weed competition at the lowest possible limit and provided almost weed free environment. Differences in crops yield were attributed to differences in weed control more the weeds present in treatment lesser will be the grain yield. It may probably the reason for higher yield in interculturing + two hand weeding at $20 \& 40$ DAS followed by aplication of imazamox 35\% + imazethapyr $35 \%$ (Pre mix) $70 \mathrm{~g} \mathrm{ha}^{-1} \mathrm{PoE} f b \mathrm{IC}+\mathrm{HW}$ at 
30 DAS and imazethapyr $75 \mathrm{~g} \mathrm{ha}^{-1} \mathrm{PoE} f b$ IC + HW at 30 DAS. Similar result was opined by Kushwah and Vyas (2005), Habimana et al., (2013) and Mishra et al., (2013) in soybean. Upadhyay et al., (2013), Rao et al., (2015) and Reddy et al., (2016) in pigeonpea. Jadhav (2015) in soybean-pigeonpea. The yield components like number of pods plant ${ }^{-1}$ and 100 seed weight are important in determining the crops yield potential. The crop weed competition in weedy check reduced the yield components in soybean and pigeonpea and thereby cause significant reduction in grain yield.

\section{Stover yield $\left(\mathrm{kg} \mathrm{ha}^{-1}\right)$}

Data regarding stover yield $\left(\mathrm{kg} \mathrm{ha}^{-1}\right)$ of soybean and pigeonpea crop was recorded as influenced by weed management practices are presented in (Table 1). The higher stover yield of soybean and pigeonpea (1525 and $1709 \mathrm{~kg} \mathrm{ha}^{-1}$ ) was recorded under treatment $\mathrm{T}_{9}(\mathrm{IC}+\mathrm{HW}$ at $20 \& 40 \mathrm{DAS})$, which was statistically at par with treatment $\mathrm{T}_{7}$ (imazamox 35\% + imazethapyr 35\% (Pre mix) $70 \mathrm{~g} \mathrm{ha}^{-1}$ PoE $f b$ IC + HW at 30 DAS), except all other treatments. Significantly lowest stover yield of soybean and pigeonpea (95 and $591 \mathrm{~kg} \mathrm{ha}^{-1}$ ) was recorded by the treatment $\mathrm{T}_{10}$ (weedy check). Increase in stover yield is directly related with increase in vegetative growth of the plant. The stover yield of soybean was affected significantly whereas, pigeonpea stover yield was significantly affected by weed control treatments. In both crop interculturing + hand weeding at $20 \& 40$ DAS was recorded higher stover yield and followed by application of imazamox 35\% + imazethapyr 35\% (Pre mix) $70 \mathrm{~g} \mathrm{ha}^{-1} \mathrm{PoE} f b$ IC $+\mathrm{HW}$ at 30 DAS. The lowest stover yield per hectare was recorded in weedy check. Similar result was opined by Kushwah and Vyas (2005), Habimana et al., (2013) and Mishra et al., (2013) in soybean. Upadhyay et al., (2013), Rao et al., (2015) and Reddy et al., (2016) in pigeonpea. Jadhav (2015) in soybean-pigeonpea.

\section{Soybean equivalent yield}

Data regarding equivalent seed and stover yield $\left(\mathrm{kg} \mathrm{ha}^{-1}\right)$ of soybean was recorded as influenced by weed management practices are presented in (Table 1 and 2).The higher equivalent seed and stover yield of $(3641 \mathrm{~kg}$ $\mathrm{ha}^{-1}$ and $4944 \mathrm{~kg} \mathrm{ha}^{-1}$ ) was recorded under treatment $\mathrm{T}_{9}(\mathrm{IC}+\mathrm{HW}$ at $20 \& 40 \mathrm{DAS})$, which was statistically at par with treatment $\mathrm{T}_{7}$ (imazamox 35\% + imazethapyr 35\% (Pre mix) $70 \mathrm{~g} \mathrm{ha}^{-1} \mathrm{PoE} f b$ IC + HW at 30 DAS) (3380 kg ha ${ }^{-1}$ and $4584 \mathrm{~kg} \mathrm{ha}^{-1}$ ). Significantly lowest equivalent seed and stover yield of (986 kg ha ${ }^{-1}$ and $1277 \mathrm{~kg} \mathrm{ha}^{-1}$ ) was recorded by the treatment $\mathrm{T}_{10}$ (weedy check). The soybean-equivalent yield was worked out considering price of soybean and pigeonpea. The observation on SEY kg ha-1 indicated that significant difference due to various treatments. Interculturing + hand weeding at 20 \& 40 DAS recorded significantly higher SEY than all other treatments except application of imazamox 35\% + imazethapyr $35 \%$ (Pre mix) $70 \mathrm{~g} \mathrm{ha}^{-1} \mathrm{PoE} f b$ IC + HW at 30 DAS. The lowest SEY recorded in weedy check. Similar result was reported by Kushwah and Vyas (2005) in soybean. Jadhav (2015) and Ilhe et al., (2017) in soybeanpigeonpea.

\section{Economics}

Data on economics of the different treatments presented in (Table 2) indicated maximum gross return and net return (114186 and $59999 \mathrm{ha}^{-1}$ ) was achieved under treatment $\mathrm{T}_{9}$ $\left(\mathrm{IC}+\mathrm{HW}\right.$ at $20 \& 40$ DAS) and $\mathrm{T}_{7}$ (imazamox 35\% + imazethapyr 35\% (Pre mix) $70 \mathrm{~g} \mathrm{ha}^{-1}$ PoE $f b$ IC + HW at 30 DAS) (105971 and $56351 \mathrm{ha}^{-1}$ ). The benefit cost ratio was also higher in $\mathrm{T}_{7}$ (imazamox $35 \%+$ imazethapyr 35\% (Pre mix) $70 \mathrm{~g} \mathrm{ha}^{-1} \mathrm{PoE} f b$ 
$\mathrm{IC}+\mathrm{HW}$ at $30 \mathrm{DAS}$ ) (2.14) followed by treatment $\mathrm{T}_{9}(\mathrm{IC}+\mathrm{HW}$ at $20 \& 40 \mathrm{DAS})$ (2.11). The treatment $\mathrm{T}_{10}$ (weedy check) had lowest benefit cost ratio (0.75) due to poor yield in this treatment. The results are in conformity with the results of Mishra et al., (2013), Upadhyay et al., (2012) and Moghal et al., (2014).

\section{Effect of applied herbicides on succeeding crops}

Data pertaining to germination (\%), plant height/shoot length $(\mathrm{cm})$,dry matter accumulation $\left(\mathrm{g} \mathrm{plant}^{-1}\right)$ and root length $(\mathrm{cm})$ of succeeding maize, pearl millet and cucumber crops revealed that did not differ significantly due to residual effect of herbicides applied in preceding crop (Table $3)$. This indicated that herbicides was applied in preceding crop had no adverse effect on germination of succeeding crops. These findings are in confirmation with findings of Sangeetha et al., (2012) and Patel et al., $\left(2016_{a}\right)$.

From the results of field experimentation, it can be concluded that weeds could be managed by adopting weed management practices interculturing + hand weeding at 20 $\& 40$ DAS or post - emergence application of imazamox 35\% + imazethapyr 35\% (Pre mix) $70 \mathrm{~g} \mathrm{ha}^{-1} \mathrm{PoE} f b$ interculturing + hand weeding at 30 DAS was recorded higher growth, growth and yield attributes, soybean equivalent yield, gross returns, net returns, benefit cost ratio and none of the herbicides showed adverse effect on succeeding crops viz., maize, pearl millet and cucumber grown after harvest of soybean and pigeonpea without disturbing soil.

\section{References}

Ali M. Weed suppressing ability and productivity of short duration legumes intercropped with pigeonpea under rainfed conditions. Tropical Pest Management. 1992; 34(4): 384-387.

Bhan VM. Weeds associated with soybean and their control. Soybean production, protection and utilization. University of Illinois Urbana (Intsoy Ser.6).1975; pp. 147-156.

Habimana S, Murthy KNK, Shankaralingappa BC, Sanjay MT and Ramachandra C. Efficiency and economics of weed control with pre and post emergence herbicides in soybean. Asian Journal of Plant Science and Research.2013; 3(4): 18-20.

Ilhe SS, Patil HM, Patil DB, Pawar PP and Wadile SC. Integrated weed management in soybean + pigeonpea intercropping system under rainfed. 2017; Department of Agronomy, Punjab Agricultural University, Ludhiana, Punjab, India $25^{\text {th }}$ Asian-Pacific Weed Science Society Conference on "Weed Science for Sustainable Agriculture, Environment and Biodiversity".

Jadhav AS. Performance of post emergence weedicide in soybean + pigeonpea Intercropping. Journal of Agriculture and Veterinary Science.2015; 8(7):6162.

Kurchania SP, Rathi GS, Bhalla CS and Mathew R. (2001). Bio efficiency of Post emergence herbicides for weed control in soybean (Glycine max). Indian Journal of Weed Science. 2001; 33(1 \& 2): 34-37.

Kushwah SS and Vyas MD. Herbicidal weed control in soybean (Glycine max). Indian Journal of Agronomy. 2005; 50(3): 225227.

Mishra P, Singh Harvir, BabuSubhash and Pal Suresh. (2013). Bioefficacy of some early post emergence herbicides in soybean. Annals of Agricultural Research. 2013; 24(4): 970- 972.

Moghal HS, Kalegore NK and Kadam SR. 
Study of different weed control treatment on economics of soybean. In: Proceeding Biennial Conference of Indian Society of Weed Science on "Emerging Challenges in Weed Management". February 15-17, 2014. Directorate of Weed Science Research, Jabalpur, Madhya Pradesh, India. p 172.

Patel BD, Chaudhari DD, Patel RB, Patel VJ and Darji VB. Comparative efficacy of different herbicides on Bt. cotton and their residual effect on succeeding crops. Research on Crops. 2016 a; 17 (1): 106-111.

Prachand S, Kubde KJ and Bankar S. Effect of chemical weed control on weed parameters, growth, yield attributes, yield and economics in soybean (Glycine max). American- Eurasian Journal of Agricultural \& Environmental Science. 2014; 14(8): 698-701.

Reddy AS, Rao PV and Rao YK. Response of IWM on growth and yield of pigeonpea. International Journal of current microbiology and applied sciences. 2016; 5(3):610-616.

Sangeetha C, Chinnusamy C and Prabhakaran NK. Early post-emergence herbicides for weed control in soybean. Indian Journal of Weed Science. 2013; 45(2):
140-142.

Sangeetha C, Chinnusamy $\mathrm{C}$ and Prabhakaran NK. Efficacy of imazethapyr on productivity of soybean and its residual effect on succeeding crops. Indian Journal of Weed Science. 2012; 44(2): 135-138.

Sobney JS, Sindhu MS, Maran RS and Kumar $\mathrm{K}$. Intercropping as a system to manage. Weed Journal of Research PAV.1989; 26(4); 563-568.

Talnikar AS, Kadam GL, Karande DR and Jogdand PB. Integrated weed management in pigeonpea [Cajanus cajan (L.) Millsp.]. International Journal of Agricultural Sciences. 2008; 4(1): 363-370.

Upadhyay VB, Singh A and Rawat A. Efficacy of early post-emergence herbicides against associated weeds in soybean. Indian Journal of Weed Science. 2012; 44(4): 73-75.

Venkat Rao P, Subbarami Reddy A. and Koteshwara Rao Y. Effect of integrated weed management practices on growth and yield of pigeonpea. International Journal of Plant, Animal and Environmental Science. 2015; 5: 124127.

\section{How to cite this article:}

Hajari, R. V. and Patel, G. J. 2020. Effect of Different Weed Management Practices on Growth and Yield in Soybean Pigeonpea Intercropping System and its Residual Effect on Succeeding Crops. Int.J.Curr.Microbiol.App.Sci. 9(09): 2328-2337. doi: https://doi.org/10.20546/ijcmas.2020.909.291 